Revista Eureka sobre Enseñanza y Divulgación de las Ciencias

ISSN: 1697-011X

revista.eureka@uca.es

Universidad de Cádiz

España

\title{
¿Qué evalúan las preguntas sobre división celular en las pruebas de acceso a la universidad?
}

Esquivel-Martín, Tamara; Pérez-Martín, José Manuel; Bravo-Torija, Beatriz

¿Qué evalúan las preguntas sobre división celular en las pruebas de acceso a la universidad?

Revista Eureka sobre Enseñanza y Divulgación de las Ciencias, vol. 19, núm. 1, 2022

Universidad de Cádiz, España

Disponible en: https://www.redalyc.org/articulo.oa?id=92068491017

DOI: https://doi.org/10.25267/Rev_Eureka_ensen_divulg_cienc.2022.v19.i1.1104 


\section{¿Qué evalúan las preguntas sobre división celular en las pruebas de acceso a la universidad?}

What do the questions on cell division in the university entrance exams assess?

Tamara Esquivel-Martín

Departamento de Didácticas Especificas (Didáctica de las Ciencias Experimentales) de la Facultad de Formación de Profesorado y Educación de la Universidad Autónoma de Madrid, España

tamara.esquivel@uam.es

iD https://orcid.org/0000-0002-0739-9099

José Manuel Pérez-Martín

Departamento de Didácticas Especificas (Didáctica de las

Ciencias Experimentales) de la Facultad de Formación de

Profesorado y Educación de la Universidad Autónoma de

Madrid, España

josemanuel.perez@uam.es

iD https://orcid.org/0000-0002-0658-9050

Beatriz Bravo-Torija

Departamento de Didácticas Específicas (Didáctica de las

Ciencias Experimentales) de la Facultad de Formación de

Profesorado y Educación de la Universidad Autónoma de

Madrid, España

beatriz.bravo@uam.es

iD https://orcid.org/0000-0001-6236-6807
DOI: https://doi.org/10.25267/

Rev_Eureka_ensen_divulg_cienc.2022.v19.i1.1104

Redalyc: https://www.redalyc.org/articulo.oa?

$\mathrm{id}=92068491017$

Recepción: 28 Abril 2021

Revisado: 20 Mayo 2021

Aprobación: 15 Julio 2021

\section{ReSUMEN:}

La escolarización debería alfabetizar científicamente a la ciudadanía para tomar decisiones con criterio sobre cuestiones sociocientíficas, como el uso de pseudoterapias para tratar el cáncer. Por ello, se necesita conocer la forma de evaluar lo aprendido en todas las etapas educativas. Sin embargo, apenas hay estudios que consideren cómo se evalúa el aprendizaje de Biología adquirido durante el Bachillerato en las pruebas de acceso a la universidad (EvAU). De manera que, en este trabajo, se analiza la demanda cognitiva requerida para responder las 188 preguntas sobre un proceso biológico tan relevante como la división celular, que han sido formuladas en los exámenes EvAU de Biología de la Comunidad de Madrid desde 2002 hasta 2019; distinguiendo entre preguntas de producto, de relato y de proceso. Los resultados muestran un predominio de las preguntas de relato y de producto, con menor demanda cognitiva, viéndose una clara tendencia a aumentar las segundas hasta 2016. Además, algunos enunciados se repiten, centrándose principalmente en: mitosis, células animales, dinámica cromosómica o etapas como la anafase; obviando estructuras y momentos del proceso importantes, como el huso acromático o la prometafase. Se discuten posibles mejoras como la inclusión de preguntas de proceso que demanden tomar decisiones.

Palabras Clave: Demanda cognitiva, Formulación de preguntas, Mitosis, Meiosis, Pruebas de acceso a la universidad.

\section{ABstRACT:}

Schooling should make citizens scientifically literate in order to make informed decisions on socio-scientific issues, such as the use of pseudo-therapies to treat cancer. Therefore, it is necessary to know how learning is being assessed at all educational stages. 
However, there are hardly any studies that consider how the learning of Biology acquired during the Baccalaureate is evaluated in the university entrance exams (EvAU). Thus, in this paper, we analyse the cognitive demand required to answer the 188 questions on a biological process as relevant as cell division, which have been asked in the EvAU Biology exams of the Community of Madrid from 2002 to 2019; distinguishing among product, narrative and process questions. The results show a predominance of narrative and product questions, with lower cognitive demand, with a clear trend towards an increase in product questions until 2016. In addition, some statements are repeated and questions tend to focus on: mitosis, animal cells, chromosome dynamics or stages such as anaphase; ignoring other important structures and moments of the process such as the achromatic spindle or the prometaphase. Possible improvements are discussed, such as the inclusion of process questions that require decision making.

KEYWORDS: Cognitive demand, Mitosis, Meiosis, Question formulation, University entrance exams.

\section{INTRODUCCIÓN}

No todo lo que leemos o vemos en los medios de comunicación es cierto (Lederman y Lederman 2016). Según el II Estudio sobre Bulos en Salud (Mateos y Llobet 2019), el cáncer es una de las enfermedades sobre la que se difunden más noticias falsas (fake news) en las redes sociales e Internet, tanto por la alarma que genera en la población, como por el desconocimiento sobre su fisiopatología, relacionada con la división celular. Ante esta pandemia global, la mejor vacuna es el fact-checking, o proceso de verificación de las informaciones en la era de la posverdad (Alonso 2019). Para ello, se necesita estar alfabetizado científica, lingüística y mediáticamente (NRC 2013). Por ejemplo, para poder juzgar el rigor de una noticia sobre un posible tratamiento antimitótico, es preciso identificar y comprender la información relevante que contenga, e interpretar si el tratamiento propuesto es capaz de mitigar las causas fisiopatológicas del cáncer, a la luz de los conocimientos previos sobre división celular. Concretamente, entender el modelo de mitosis y las causas de la proliferación celular descontrolada, manejando ideas como los puntos de control del ciclo celular, ayudaría a distinguir tratamientos farmacológicos que realmente permiten frenar el avance del cáncer, de pseudoterapias sin fundamento que resultan perjudiciales para la salud (Johnson, Park, Gross y James 2018).

La educación científica formal debería centrarse en la adquisición y desarrollo de este tipo de conocimientos y destrezas, promoviendo que los estudiantes alcancen un nivel de dominio suficiente al finalizar su escolarización (NRC 2013; Real Decreto 1105/2014; OECD 2019), y estén preparados para enfrentarse a problemas durante su vida cotidiana o su desempeño profesional (Vázquez y Manassero 2019).

En concreto, si nos centramos en lo que se espera que el alumnado de Bachillerato sepa y sepa hacer en relación con la división celular, a nivel conceptual, deberían ser capaces de:

«Identificar las fases del ciclo celular, explicitando los principales procesos que ocurren en ellas; reconocer en distintas micrografías y esquemas [de células animales y vegetales] las diversas fases de la mitosis y la meiosis, indicando los acontecimientos básicos que se producen en ellas; establecer las analogías y diferencias más significativas entre mitosis y meiosis; o resumir la relación de la meiosis con la reproducción sexual, el aumento de la variabilidad genética y la posibilidad de evolución de las especies» (Real Decreto 1105/2014, p.220).

Igualmente, deberían estar preparados para: utilizar conceptos de forma cualitativa al explicar situaciones de su entorno; interpretar información científica y utilizarla para argumentar y formarse una opinión propia; o expresarse adecuadamente al tomar decisiones (Balastegui, Palomar y Solbes 2020). En este sentido, llama la atención que en el currículo de Bachillerato se pierda el bloque de "Proyecto de investigación" que incluyen los cursos de $1^{\circ}, 3^{\circ}$ y $4^{\circ}$ de ESO (Real Decreto $1105 / 2014$ ), donde se incide en la relevancia de desarrollar, integrar y aplicar las destrezas propias de los métodos de la ciencia. Tras finalizar esta etapa educativa, la vía más común de acceso a carreras universitarias en España requiere, además de disponer del título de Bachiller, superar la Evaluación para el Acceso a la Universidad (EvAU, antigua "Selectividad"), que es diferente en cada Comunidad Autónoma. Consiste en una serie de exámenes de distintas materias que pretenden evaluar si la madurez académica, conocimientos y competencias de los alumnos aspirantes a cursar grados universitarios de cualquier rama de conocimiento son suficientes (Revesado 2018). 
En este marco, nos planteamos, ¿en la EvAU se examina y valida realmente la adquisición de destrezas como el uso de datos al argumentar sobre problemas relacionados con la división celular? ¿demanda que el alumnado aplique su conocimiento en contextos como la lectura de una noticia o la interpretación de imágenes?

En el caso de la asignatura de Biología, apenas se han hallado trabajos que examinen si las preguntas formuladas a los estudiantes permiten conocer si han alcanzado un nivel adecuado de alfabetización científica antes de entrar en la universidad. En este estudio, nos centramos en el contenido de división celular por ser un tema recurrente durante la educación formal, dada la relevancia de su sentido biológico, y porque, anualmente, se incluyen preguntas sobre mitosis y meiosis en los exámenes EvAU. Concretamente, analizamos el tipo de preguntas sobre división celular que han sido formuladas en la EvAU de la Comunidad de Madrid (2002-19), tratando de dar respuesta a las siguientes preguntas de investigación:

$\cdot$ ¿Qué demanda cognitiva predomina en las preguntas de EvAU sobre división celular?

- ¿Cómo ha sido la evolución de las preguntas, atendiendo a su demanda cognitiva, desde 2002?

- ¿Se repiten preguntas a lo largo del tiempo?

- ¿Qué contenidos sobre división celular se abordan con más frecuencia en ellas?

\section{MARCo TEÓRICo}

\section{Alfabetización científica}

Durante la educación formal, la enseñanza de las ciencias se considera vital en la mayoría de los países del mundo (OECD 2019). En España, las asignaturas del ámbito científico-tecnológico tienen carácter obligatorio hasta los 16 años (Vázquez, Acevedo y Manassero 2005), edad a la que se espera que el alumnado esté alfabetizado científicamente. Este concepto de alfabetización científica, más allá de ser un eslogan pegadizo en educación (Yore 2012), es controvertido, y su definición ha ido cambiando con el paso del tiempo, lo que ha repercutido en las políticas y prácticas educativas (Siarova Sternadel y Szőnyi 2019).

Inicialmente, se entendía que su finalidad era propedéutica: comprender conceptos y desarrollar destrezas para poder cursar carreras científicas y desempeñar profesiones relacionadas con la ciencia (Vázquez et al. 2005). Con el tiempo, fue adquiriendo relevancia el objetivo social de la alfabetización científica, acercando la ciencia a la cotidianidad del alumnado, a fin de constituir ciudadanos informados, libres y responsables (Pérez-Martín 2018; Rosales, Rodríguez y Romero-Ariza 2020). Así, los enfoques actuales son más amplios (ciencia para todos) y consideran que estar alfabetizado también implica ser capaz de (Figura 1): comunicarse eficazmente (alfabetización fundamental); identificar y comprender problemas científicos (conocimientos y competencias cientificas básicas); aplicar el conocimiento y juzgar críticamente la información disponible al tomar decisiones o resolver problemas sobre cuestiones científicas, políticas, ambientales, tecnológicas y/o sociales (comprensión cientifica contextual y pensamiento critico); y, en el mejor de los casos, tomar acciones y alcanzar un compromiso activo con la sociedad (compromiso) (Siarova et al. 2019). Esto hace que su adquisición siga siendo uno de los mayores retos educativos, lo que lleva, inevitablemente, a pensar en su evaluación. 


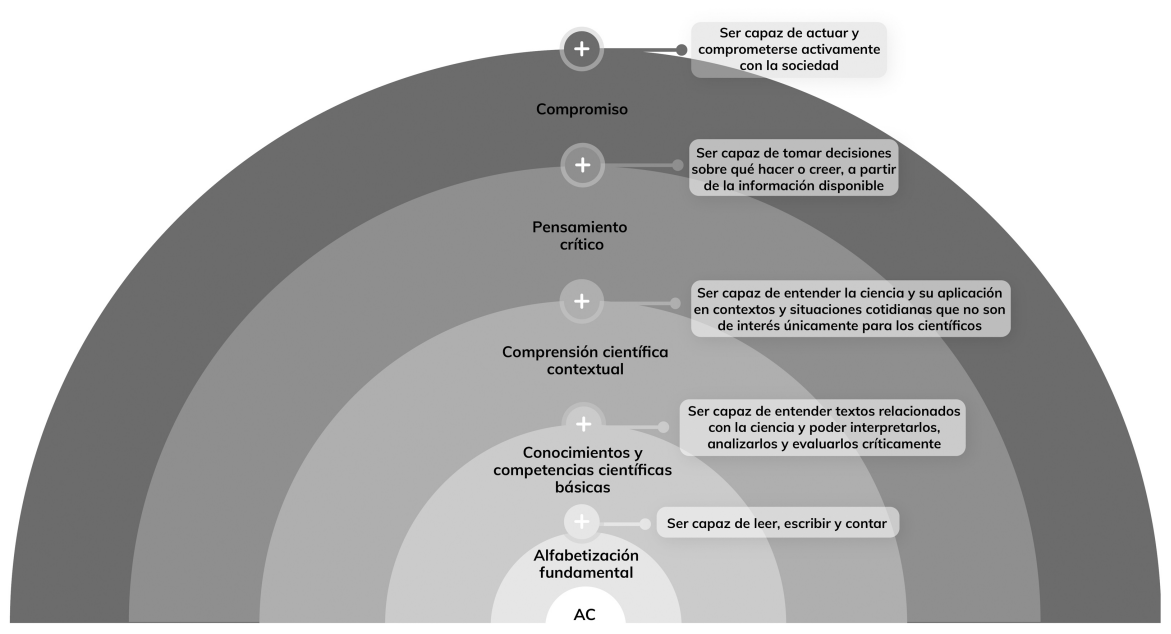

FIGURA 1

Cinco elementos clave para el desarrollo de una adecuada alfabetización científica (AC). Elaboración propia basada en Siarova et al. (2019).

\section{La importancia de las preguntas al enseñar, aprender y evaluar ciencias}

Según el currículo de ciencias preuniversitario, la Biología de segundo de Bachillerato debería capacitar a los alumnos para plantear hipótesis, hacer predicciones o resolver problemas (Real Decreto 1105/2014); todas ellas destrezas relacionadas con la formulación de preguntas y respuestas (NRC 2013; Roca, Márquez y Sanmartí 2013). Pero, en las aulas, las preguntas normalmente surgen de los docentes (Bravo-Torija, Gil, Martínez y Embid 2016). Menos frecuentes son los entornos en los que se permite a los estudiantes plantear sus propias preguntas como forma de aprendizaje (García-González y Furman 2014). No sorprende entonces que Roca et al. (2013) analicen las dificultades del alumnado para formular buenas preguntas científicas.

Por tanto, trabajar la habilidad de cuestionar y cuestionarse a través del discurso es esencial durante el aprendizaje de Biología (Bravo-Torija et al. 2016; NRC 2013); y más aún cuando se abordan contenidos a escala microscópica, como la división celular, que suscitan muchos interrogantes por percibirlos tan alejados de nuestra vida cotidiana (Esquivel-Martín, Pérez-Martín y Bravo-Torija 2021). Tanto es así que a los alumnos les cuesta modelizar la mitosis y la meiosis como procesos continuos; desconocen cómo evolucionan algunas de las estructuras que intervienen; y tienen problemas para identificar sus diferentes etapas en imágenes reales, sin conseguir relacionarlas con las representaciones de referencia (Dikmenli 2010). Por ello, desde la investigación educativa se están lanzando propuestas que pretenden mejorar la enseñanzaaprendizaje de la división celular durante la ESO. En ellas se incide en la necesidad de abordar los contenidos en sesiones que partan de preguntas que promuevan el desarrollo de destrezas científico-comunicativas como la representación y el uso de modelos, la descripción de fenómenos o el establecimiento de conclusiones a partir de datos (Esquivel-Martín et al. 2021), y donde se usen micrografías o animaciones realistas (Occelli, García, Valeiras y Willging 2017; Pérez-Martín y Aquilino 2015).

$\mathrm{Al}$ igual que es importante que los alumnos aprendan a formular preguntas científicas, también es relevante conocer cómo son empleadas las preguntas para evaluarles; valorando si existe coherencia entre el contenido que se enseña, el método seguido para ello y la forma en que se evalúa (Jin, Mikeska, Hokayem y Mavronikolas 2019). Por ello, el análisis de preguntas de evaluación ha sido objeto de diversas investigaciones, tanto en libros de texto (Pérez-Martín, Calurano, Martín-Aguilar, Esquivel-Martín y Bravo-Torija 2019), como en exámenes e instrumentos de evaluación escolares (Miralles, Gómez y Sánchez 2014). Pero también en pruebas oficiales estandarizadas; así, algunos autores han analizado la tipología de preguntas existente en 
evaluaciones externas como el Programa para la Evaluación Internacional de Alumnos (PISA) (Rosales et al. 2020) o en los exámenes de acceso a la universidad de asignaturas como Química (Franco-Mariscal, Oliva, y Gil 2015). En cuanto a Biología, desde el estudio de Menoyo (2003), apenas hemos encontrado trabajos donde se analicen las preguntas de selectividad; y mucho menos las centradas en un contenido tan recurrente y relevante como la división celular.

En los trabajos anteriores, las preguntas se clasifican atendiendo a los objetivos del análisis, pudiendo considerarse el tipo de pregunta (abierta/cerrada, etc.) o el conocimiento que se evalúa (conceptual, procedimental, epistémico) (OECD 2019). En este estudio, nos centramos en la demanda cognitiva requerida para responder preguntas sobre división celular, para lo cual no existe una clasificación única (universal) que podamos utilizar en asignaturas de ciencias. Aunque todas las que existen guardan relación, la tarea de comparar categorizaciones resulta ardua en ocasiones, ya que cada autor se basa en aquellas que responden a sus intereses y que más se ajustan a su área de conocimiento, revisándolas y reajustándolas si lo consideran necesario. En este sentido, una de las primeras taxonomías fue la desarrollada por Bloom (1956), quien empleó seis categorías, ordenadas de menor a mayor complejidad, en función de las destrezas requeridas para responder preguntas, lo que se relaciona precisamente con la dimensión cognitiva del aprendizaje: a. conocimiento; b. comprensión; c. aplicación; d. análisis; e. sintesis; y f. evaluación.

Posteriormente, se generaron nuevas categorizaciones. Si nos centramos en preguntas de evaluación en asignaturas de Ciencias Experimentales, en Química suele utilizarse la clasificación de Zoller (1995): destrezas cognitivas de bajo (LOCS) y alto nivel (HOCS), que requieren recordar y aplicar conocimientos en situaciones y contextos familiares; o aplicar, analizar, sintetizar y evaluar conocimientos en situaciones desconocidas, respectivamente; preguntas algoritmicas, que demandan emplear un conjunto de procedimientos memorizados para responderlas; $y$ preguntas conceptuales, que precisan manejar conceptos subyacentes de las teorías científicas básicas. Más tarde, Smith, Nakhleh y Bretz (2010) sintetizaron varias clasificaciones, entre ellas la de Zoller (1995), distinguiendo entre preguntas de definición, que demandan recordar, comprender, aplicar o reconocer una definición; algoritmicas, que requieren utilizar información o procesos memorizados; y conceptuales, que solicitan realizar algún tipo de análisis no algorítmico. Entendían que estas categorías permiten clasificar cualquier problema de Química general, al considerar tanto los datos aportados por la pregunta, como el razonamiento requerido para responderla. Para analizar las preguntas de Química en los exámenes de acceso a la universidad, Franco-Mariscal et al. (2015) se basaron en la clasificación de Smith. et al. (2010), incluidas las subcategorías de estos autores. Por ejemplo, dividiendo las preguntas de definición en: a. recordar, comprender o aplicar una definición, y b. reconocer una definición. Se observa, por tanto, una tendencia a la reducción de categorías de preguntas en función de su demanda cognitiva, desde las seis propuestas por Bloom (1956), hasta las tres de Smith et al. (2010). Esto hace que deban detallarse más las características que han de reunir las cuestiones para ser incorporadas en unas categorías u otras, lo que comúnmente deriva en la generación de subcategorías que atiendan a las particularidades de cada materia.

En Biología, el marco de referencia es escaso. Trabajos como el de Quse y Masullo (2017) analizan la exigencia cognitiva de las preguntas del Operativo Nacional de Evaluación 2016 en Argentina, clasificándolas en los niveles de dificultad alto, medio y bajo que se usan en las pruebas PISA. En la tesis doctoral de Ferrés-Gurt (2017), se analizan las preguntas conceptuales y de indagación de las pruebas EvAU de Cataluña (2011-15), considerando su grado de apertura o con qué habilidades de la competencia científica se relacionan. En la misma línea, la clasificación de Furman, Poenitz y Podestá (2012), divide las preguntas de cualquier examen en dos tipos, atendiendo a la dimensión de las ciencias que se evalúa. Es decir, si se entiende como conjunto de conocimientos (ciencia como producto), incluyendo preguntas fundamentalmente cerradas y relacionadas con conceptos, hechos y datos; o si se evalúa cómo se construye la ciencia y sus implicaciones (ciencia como proceso), incluyendo preguntas abiertas que demandan poner en práctica destrezas científicas como clasificar, formular hipótesis, identificar variables o analizar datos. Asimismo, cada dimensión se 
subcategoriza atendiendo al tipo de operación cognitiva requerida para responder, dividiendo las preguntas de producto en: terminológicas, que indagan sobre términos y definiciones; descriptivas, que demandan enumerar o representar las características de objetos o situaciones naturales; o explicativas, que requieren formular el proceso o mecanismo que origina cierto fenómeno. Y las de proceso en: orientadas a procesos lógicos (formular hipótesis, interpretar datos, clasificar, etc.), u orientadas a procesos técnicos (usar instrumentos o material de laboratorio). En este sentido, consideramos que la falta de un criterio unánime para analizar preguntas en Biología, debería llevarnos, como ocurre en Química, a emplear una clasificación sencilla (2-3 categorías generales), para atender después a la idiosincrasia de la asignatura generando subcategorías.

\section{Metodología}

En este estudio descriptivo, se analiza el contenido de 188 preguntas sobre división celular extraídas de 68 exámenes EvAU de Biología de la Comunidad de Madrid. El periodo de estudio comprende 38 convocatorias desde 2002 hasta 2019. Los exámenes pueden consultarse en el repositorio de la Universidad Autónoma de Madrid (http://bit.ly/3s85qdV).

Para analizar la demanda cognitiva de las preguntas nos basamos en la clasificación propuesta por Furman et al. (2012), a la que incorporamos una categoría intermedia denominada preguntas de relato. En ella, se incluyen las preguntas que demandan al alumnado explicar el contenido sin aplicar los conocimientos a un caso concreto, sintetizando la información transmitida por los docentes y los recursos educativos utilizados durante el proceso de enseñanza. Por ello, consideramos que se encuentran en un punto intermedio entre las preguntas de proceso, que son aquellas que, para responderlas, requieren razonar o aplicar el conocimiento sobre una situación particular diferente a la del libro de texto; y las preguntas de producto, que solo demandan reproducir datos, definiciones concretas o esquemas básicos.

Por ejemplo, una pregunta de relato sería aquella en la que haya que construir un relato escrito o dibujado para describir alguna etapa del ciclo celular (incluida la mitosis), o de la meiosis (p. ej. junio 2012, 4.b: explique el proceso de citocinesis en una célula animal y en una vegetal); pasando por determinados eventos principales que son los que, tradicionalmente, una pregunta de producto demandaría enumerar (p.ej. septiembre 2012, 4.b: indique cuatro de los principales acontecimientos que tienen lugar durante la telofase mitótica). Para responder a esta clase de preguntas, los estudiantes deberían integrar las ideas del modelo de división celular, organizando con sus propias palabras su respuesta.

En resumen, nuestra herramienta de análisis distingue tres categorías en función de la demanda cognitiva de las preguntas sobre división celular, que, ordenadas de menor a mayor, son: preguntas de producto, preguntas de relato y preguntas de proceso. Además, dentro de cada categoría, los autores han generado subcategorías en orden creciente de complejidad, surgidas de la interacción con los datos (ver Tabla 1).

Para determinar la fiabilidad del proceso de categorización (O’Connor y Joffe 2020), los autores analizaron las preguntas de forma independiente, tras acordar las categorías y los criterios de inclusión de cada una. Posteriormente, se compararon los resultados. A pesar de darse un alto consenso en la mayoría de casos, se detectaron categorías ambiguas o desiertas. Por ello, algunas se recodificaron, con el fin de aclararlas, y otras se eliminaron. Por ejemplo, inicialmente, se consideró una subcategoría de "toma de decisiones" dentro de la categoría de preguntas de proceso, pero, al no existir preguntas que pudieran incluirse en ella, fue suprimida. Finalmente, los autores repitieron individualmente el análisis de las preguntas, alcanzando un porcentaje de coincidencia del 91,49\% (172/188 casos). Las diferencias se discutieron hasta llegar al consenso total. 
TABLA 1

Ejemplos de la categorización empleada por los autores para clasificar las preguntas de EvAU sobre división celular.

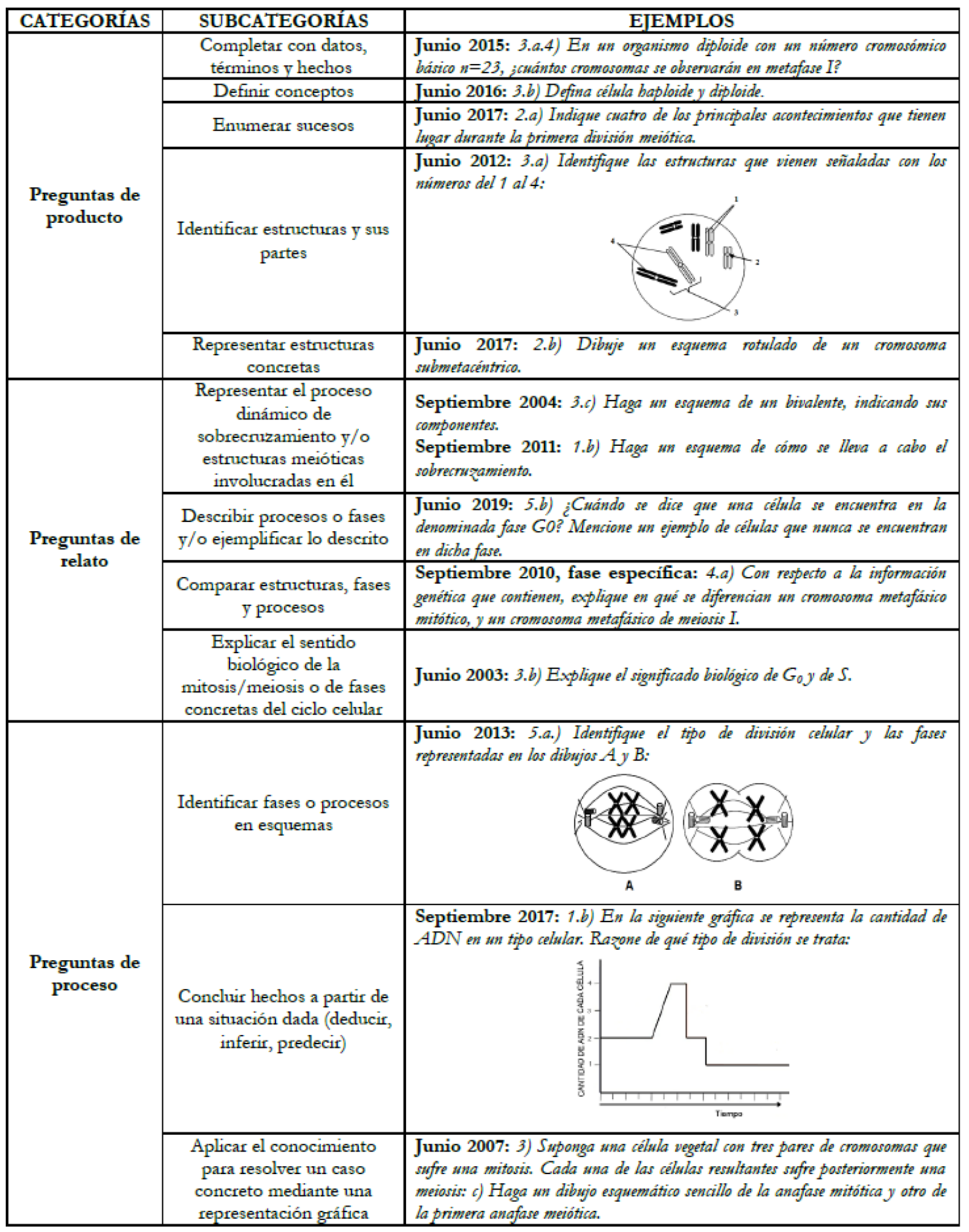

Para responder a las preguntas de investigación de este trabajo, tras clasificar la muestra de preguntas estudiada en función de su demanda cognitiva, se analiza la frecuencia de aparición de cada categoría y subcategoría en los exámenes, y se comprueba si existen diferencias significativas entre estas frecuencias a lo 
largo del tiempo mediante la prueba Chi-cuadrado (Microsoft Excel 2016). Además, para profundizar en el análisis, también se explora si existen enunciados repetidos entre convocatorias, y qué contenidos sobre división celular se abordan mayoritariamente en las preguntas.

\section{RESULTADOS Y DISCUSióN}

En relación con la demanda cognitiva que ha predominado en las preguntas sobre división celular de la EvAU, los resultados muestran que este contenido se ha evaluado tradicionalmente mediante preguntas de relato (36,70\%) y de producto (35,64\%), ambas con demandas cognitivas más bajas (72,34\%), siendo minoritarias las de proceso (27,66\%) (Tabla 2). En las frecuencias de las subcategorías encontramos notables diferencias, predominando las que requieren completar con datos (17,55\%); describir procesos y fases (17,55\%); y resolver casos concretos (12,23\%). Sin embargo, son muy pocas las que demandan identificar $(2,13 \%)$ o representar estructuras (1,60\%) involucradas en el proceso de división celular. En este sentido, parece excesivo el protagonismo de la terminología en la EvAU, con 33 preguntas que se responden completando enunciados y tablas con nombres (fase, estructura, tipo celular) (Tabla 2). Aunque el manejo de lenguaje científico riguroso es importante y, con ello, la evaluación del aprendizaje conceptual sobre división celular, no se deben desatender el resto de dimensiones de la alfabetización científica, como la capacidad de tomar decisiones (Figura 1); algo que, como muestran los resultados, ocurre en la EvAU.

TABLA 2

Frecuencias absolutas (FA) y relativas (FR) de preguntas en cada categoría y subcategoría respecto del total de preguntas analizadas (188).

\begin{tabular}{|c|c|c|c|c|c|}
\hline CATEGORÍAS & FA & FR & SUBCATEGORÍAS & FA & FR \\
\hline \multirow{5}{*}{$\begin{array}{l}\text { Preguntas de } \\
\text { producto }\end{array}$} & \multirow{5}{*}{67} & \multirow{5}{*}{$35,64 \%$} & $\begin{array}{l}\text { Completar con datos, términos y } \\
\text { hechos }\end{array}$ & 33 & $17,55 \%$ \\
\hline & & & Definir conceptos & 17 & $9,04 \%$ \\
\hline & & & Enumerar sucesos & 10 & $5,32 \%$ \\
\hline & & & Identificar estructuras y sus partes & 4 & $2,13 \%$ \\
\hline & & & Representar estructuras concretas & 3 & $1,60 \%$ \\
\hline \multirow{4}{*}{$\begin{array}{l}\text { Preguntas de } \\
\text { relato }\end{array}$} & \multirow{4}{*}{69} & \multirow{4}{*}{$36,70 \%$} & $\begin{array}{l}\text { Representar el proceso dinámico de } \\
\text { sobrecruzamiento y jo las estructuras } \\
\text { meióticas involucradas en él }\end{array}$ & 4 & $2,13 \%$ \\
\hline & & & $\begin{array}{l}\text { Describir procesos y fases y lo } \\
\text { ejemplificar lo descrito }\end{array}$ & 33 & $17,55 \%$ \\
\hline & & & Comparar estructuras, fases y procesos & 14 & $7,45 \%$ \\
\hline & & & $\begin{array}{l}\text { Explicar el sentido biológico de la } \\
\text { mitosis /meiosis o de fases concretas } \\
\text { del ciclo celular }\end{array}$ & 18 & $9,57 \%$ \\
\hline \multirow{3}{*}{$\begin{array}{l}\text { Preguntas de } \\
\text { proceso }\end{array}$} & \multirow{3}{*}{52} & \multirow{3}{*}{$27,66 \%$} & $\begin{array}{l}\text { Identificar fases o procesos en } \\
\text { esquemas }\end{array}$ & 11 & $5,85 \%$ \\
\hline & & & $\begin{array}{l}\text { Concluir hechos a partir de una } \\
\text { situación dada (deducir, inferir, } \\
\text { predecir) }\end{array}$ & 18 & $9,57 \%$ \\
\hline & & & $\begin{array}{l}\text { Aplicar el conocimiento para resolver } \\
\text { un caso concreto mediante una } \\
\text { representación gráfica }\end{array}$ & 23 & $12,23 \%$ \\
\hline
\end{tabular}

Estos hallazgos, centrados en la división celular, complementan los del estudio de Ferrés-Gurt (2017) en Cataluña, donde, en un análisis global, se habla de la tendencia a aumentar las preguntas cerradas (sin justificar, equivalentes a la categoría de producto) en los exámenes de Biología para acceder a la universidad (2011-15). Estas se centran en el conocimiento conceptual del alumnado y no en evaluar su habilidad indagadora. Asimismo, en el estudio de Franco-Mariscal et al. (2015), donde se analizan exámenes de 
selectividad de Química en Andalucía (2002-13), también predominan las preguntas algorítmicas (nivel intermedio de demanda cognitiva, equivalentes a las preguntas de relato en este trabajo), asociadas a un aprendizaje mecánico; en detrimento de las preguntas conceptuales, más exigentes por la madurez de razonamiento que requieren y que se corresponderían con las preguntas de proceso del presente estudio. Esta realidad no encajaría en un modelo ideal de evaluación que compruebe si los estudiantes han desarrollado un nivel de alfabetización científica y unas competencias profesionales suficientes para acceder a carreras como Biología, y desenvolverse con éxito al aplicar sus conocimientos científicos en contextos académicos y/ o cotidianos. Así, dentro de las competencias propuestas por Sánchez (2004) en el Libro Blanco del Título de Grado en Biología, están la capacidad tomar decisiones (p.ej. reconocer y analizar nuevos problemas y planear estrategias para solucionarlos), la interpretación de los datos obtenidos de observaciones y medidas en base a los modelos explicativos subyacentes, o la planificación del manejo de instrumental de laboratorio como el microscopio al diseñar/realizar experimentos.

Llama la atención que, en la etapa educativa anterior, también predominen las preguntas de evaluación que favorecen la reproducción del conocimiento conceptual del alumnado frente a su aplicación en contextos reales, como puede verse en el estudio de Rosales et al. (2020). Estas autoras valoran si las pruebas PISA, destinadas a evaluar la competencia científica del alumnado de 15 años, permiten determinar realmente si los estudiantes son capaces de: a) explicar los fenómenos cientificamente. b) evaluar y diseñar experimentos; y c) interpretar los datos y las pruebas cientificamente (OECD 2019). Los resultados muestran que, en la práctica, la demanda cognitiva de estas pruebas desde el año 2000 ha sido predominantemente baja (>80\%). Esto se debe a que las actividades que requieren únicamente procesos mentales como recordar (p. ej. verdadero/falso) o comprender (p. ej. explicar algún aspecto, en ocasiones apoyándose en una tabla o figura) son mayoritarias, recurriendo frecuentemente a contextos académicos, en lugar de otros más cercanos a la vida cotidiana de los estudiantes.

Con respecto a la evolución trianual de los tipos de preguntas en función de su demanda cognitiva, vemos que la distribución de las categorías entre trienios no es homogénea $\left(X^{2}=16,24 ; \mathrm{gl}=10 ; \alpha=0,1\right)$ (Tabla 3). Es de destacar que, en el último (2017-19), se ha reducido el número total de preguntas sobre división celular en la EvAU, ya que se incluyen en menos opciones de las distintas convocatorias (junio y julio/septiembre).

TABLA 3

Frecuencias absolutas de preguntas en cada categoría por trienios. Ex.: exámenes.

\begin{tabular}{|l|l|l|l|l|l|l|}
\cline { 2 - 7 } \multicolumn{1}{c|}{} & 12 ex. & 12 ex. & 14 ex. & 12 ex. & 12 ex. & 6 ex. \\
\cline { 2 - 8 } \multicolumn{1}{c|}{} & $2002-04$ & $2005-07$ & $2008-10$ & $2011-13$ & $2014-16$ & $2017-19$ \\
\hline $\begin{array}{l}\text { Preguntas } \\
\text { de } \\
\text { producto }\end{array}$ & 5 & 10 & 13 & 16 & 17 & 6 \\
\hline $\begin{array}{l}\text { Preguntas } \\
\text { de relato }\end{array}$ & 16 & 14 & 16 & 10 & 9 & 4 \\
\hline $\begin{array}{l}\text { Preguntas } \\
\text { de } \\
\text { proceso }\end{array}$ & 11 & 8 & 14 & 9 & 4 & 6 \\
\hline Total & 32 & 32 & 43 & 35 & 30 & 16 \\
\hline
\end{tabular}

Para comparar la evolución temporal del tipo de preguntas, se ha calculado su frecuencia normalizada, dividiendo la frecuencia absoluta de cada categoría en cada trienio entre el total de exámenes de ese periodo (Figura 2). Hasta 2016, se ve una clara tendencia a aumentar la ratio de preguntas de producto incluidas en cada examen, y disminuir la de preguntas de relato y de proceso. 


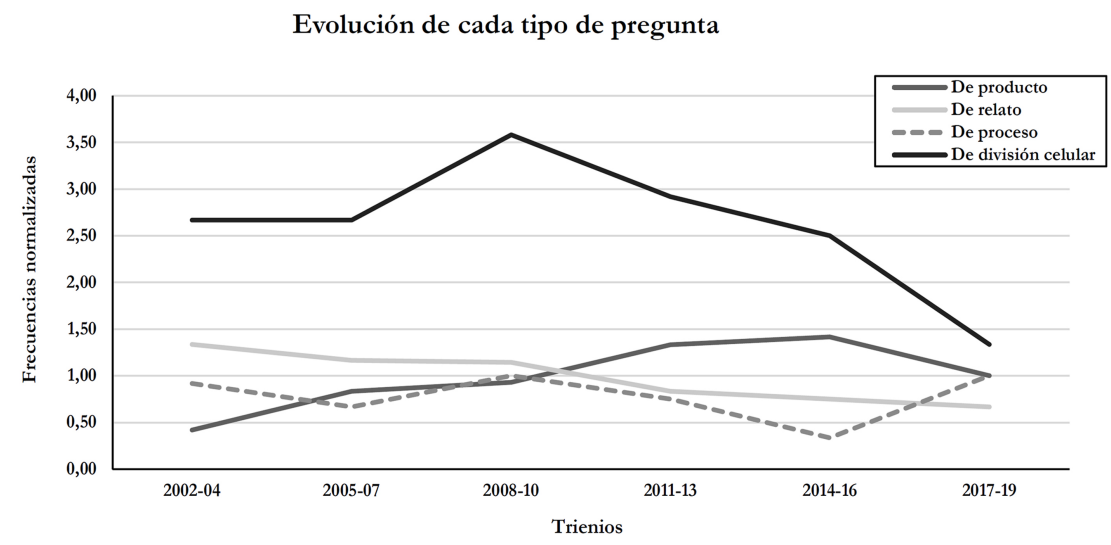

FIGURA 2

Evolución de las preguntas de cada tipo por examen, agrupadas en trienios.

$\mathrm{Si}$, en cambio, dividimos los años de la muestra atendiendo a la ley educativa vigente en cada momento (Tabla 4), encontramos que, con la Ley Orgánica General del Sistema Educativo (LOGSE), la exigencia cognitiva de las pruebas parece mayor, incluyéndose más preguntas de relato y de proceso. Esto cambia durante la Ley Orgánica de Educación (LOE), predominando las preguntas de relato y de producto. Finalmente, desde 2013 (Ley Orgánica para la Mejora de la Calidad Educativa o LOMCE), se da mayor peso a las preguntas de producto, a pesar de las recomendaciones internacionales, que abogan por el empleo de preguntas de indagación, más experimentales, y que demanden el pensamiento aplicado del alumnado (Vieira y Tenreiro-Vieira 2016). De hecho, existen diferencias significativas entre la frecuencia de los tipos de preguntas durante la LOGSE y la LOMCE $\left(X^{2}=4,605 ; \mathrm{gl}=2 ; \alpha=0,1\right)$. Parece, por tanto, que la demanda cognitiva de las preguntas ha disminuido con los cambios legislativos, posiblemente para facilitar su corrección, lo que podría repercutir en una mejor calificación en los exámenes de Biología de la EvAU.

TABLA 4

Frecuencias absolutas de preguntas en cada categoría por leyes educativas. Ex.: exámenes.

\begin{tabular}{|l|l|l|l|l|}
\cline { 2 - 5 } \multicolumn{1}{c|}{} & 16 Ex. & $30 \mathrm{Ex}$. & 22 Ex. & \\
\cline { 2 - 5 } \multicolumn{1}{c|}{} & $\begin{array}{l}\text { LOGSE } \\
\text { 2002-05 }\end{array}$ & $\begin{array}{l}\text { LOE } \\
\text { 2006-12 }\end{array}$ & $\begin{array}{l}\text { LOMCE } \\
\text { 2013-19 }\end{array}$ & Total \\
\hline $\begin{array}{l}\text { Preguntas } \\
\text { de } \\
\text { producto }\end{array}$ & 11 & 30 & 26 & 67 \\
\hline $\begin{array}{l}\text { Preguntas } \\
\text { de relato }\end{array}$ & 20 & 34 & 15 & 69 \\
\hline $\begin{array}{l}\text { Preguntas } \\
\text { de } \\
\text { proceso }\end{array}$ & 14 & 22 & 16 & 52 \\
\hline Total & 45 & 86 & 57 & 188 \\
\hline
\end{tabular}

$\mathrm{Al}$ explorar si se repiten enunciados a lo largo del tiempo en la EvAU, hallamos que, en general, ha cambiado poco la forma en que se formulan las preguntas entre ediciones consecutivas. Por ejemplo, si atendemos a la categoría de relato, desde 2002, hasta en 11 pruebas se pide a los estudiantes que expliquen la importancia biológica de la meiosis. En menos casos (3), la de la mitosis. Incluso en tres exámenes se demanda que expliquen ambos sentidos biológicos y/o los comparen. También encontramos preguntas de producto repetidas en convocatorias próximas. Como ejemplo: Dibuje un esquema rotulado de un cromosoma submetacéntrico señalando cuatro de las estructuras que lo componen (junio 2017, 2.b; septiembre 2016, 1.b). 
En cuanto a las preguntas de proceso, son varios los enunciados que demandan realizar esquemas de la anafase mitótica (junio 2014, 2.b; septiembre 2013, 4.b; septiembre 2010, fases general, 3.a y específica, 4.a; junio 2008, 4.a; septiembre 2005, 3.c; septiembre 2002, 3.a). Por su parte, los exámenes de junio 2011 (1.a), junio 2009 (4.a) o septiembre 2002 (3.c) demandan representar la anafase I o II. Finalmente, en junio 2010 (3.a, fase específica), septiembre 2007 (3.a), junio 2004 (3.b) o junio 2002 (3.b), se solicita dibujar anafases de dos tipos (mitótica y/o meióticas I y II). En todos los casos las dotaciones cromosómicas varían entre $2 n=4$ y $2 n=6$. En ocasiones se especifica el tipo celular (animal/vegetal) o los cromosomas a representar (metacéntricos y acrocéntricos). En este sentido, creemos que una sutil repetición de casos a lo largo del tiempo no tiene por qué ser considerada como algo negativo. Pero, si ocurre con excesiva frecuencia, podría contribuir a que, aunque en origen la demanda cognitiva de las preguntas sea de relato (p. ej. sentido biológico) o de proceso (p. ej. aplicación del conocimiento en situaciones concretas), con los años, pase a ser de producto, al poder memorizarse «respuestas tipo».

Con respecto al contenido sobre división celular evaluado, hay más preguntas sobre mitosis (82), que sobre meiosis (58), existiendo otras 48 que demandan conocer ambos procesos. Este aparente desequilibrio favorece al alumnado, ya que presentan más dificultades de comprensión e ideas alternativas sobre el modelo de meiosis (Dikmenli 2010), lo que se ve cuando llegan a la universidad (Rodríguez, Fradkin y Castañeda 2018). Además, llama la atención que el propio examen contenga errores, preguntando sobre meiosis con enunciados como: «Con relación al ciclo celular: [...]» (p. ej. junio 2011, 2.b; junio 2015, 3.b), ya que el ciclo celular solo incluye la mitosis. Si atendemos al tipo celular, 24 preguntas giran en torno a células animales: 19 de forma explícita, sumado a cinco enunciados que hablan de células epiteliales, especie humana o divisiones astrales. Por su parte, en 16 ocasiones se deben responder cuestiones acerca de células vegetales, mencionándolas explícitamente 13 veces; o indirectamente, hablando de divisiones anastrales (3). Esto podría deberse a que el modelo prototípico de célula animal, predominante en los esquemas de los libros de texto, parece asimilarse mejor que el de célula vegetal (Caballer y Giménez 1993). Lo anterior resulta paradójico, ya que, dada la dificultad de obtener buenas imágenes del proceso de división celular a partir de tejido animal (Domènech 2016), durante la ESO y el Bachillerato, se suele utilizar material biológico vegetal (p. ej. raíz de cebolla) para obtener preparaciones microscópicas.

$\mathrm{Si}$ atendemos a la presencia de imágenes en las pruebas, a pesar de ser esenciales para entender el modelo de división celular, solo aparecen esquemas del proceso o de las estructuras que intervienen (fundamentalmente cromosomas) en 17 preguntas. Esto dificulta caracterizar la destreza del alumnado manejando e interpretando este tipo de representaciones. Cabe destacar que, en ningún caso, se incluyen micrografías. $\mathrm{Y}$, aunque no sea objetivo directo del estudio, en algunos esquemas se han encontrado errores como la representación del huso inadecuada, que no permite distinguir los microtúbulos cinetocóricos del resto (Figura 3A); o la pérdida de cohesividad entre cromátidas hermanas anterior a la metafase (algo que no ocurre hasta dispararse anafase), representando los cromosomas como cruces (Figura 3B). Este tipo de errores también han sido detectados en imágenes de libros de texto (Pérez-Martín y Aquilino 2015) y, consecuentemente, en los dibujos de los estudiantes cuando se les demanda representar la mitosis (EsquivelMartín et al. 2021).
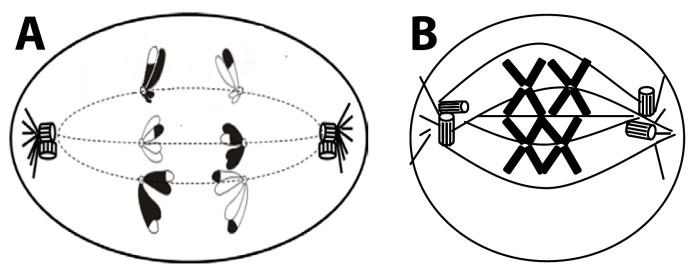

FIGURA 3

Esquemas (con errores) asociados a preguntas de EvAU en junio 2018 (A) y junio 2013 (B). 
Por otra parte, se pide a los estudiantes que representen gráficamente la división celular hasta en 30 ocasiones, requiriendo que expresen sus modelos mentales en el contexto de preguntas específicas. Normalmente se les demanda dibujar estructuras y fases concretas, perpetuando la idea de que son «fotos fijas», en lugar de promover que los alumnos reflejen la continuidad del proceso (Esquivel-Martín et al.2021; Occelli et al. 2017).

$\mathrm{Si}$ atendemos a las fases, de las 93 veces que se mencionan etapas concretas de la división celular en las preguntas, la anafase (18), la citocinesis (12) y la metafase (10) mitóticas son las que más aparecen. En el caso de la meiosis, predominan las anafases I (8) y II (9). Sin embargo, en ningún momento se mencionan la prometafase, o fases de la segunda división meiótica como profase II, metafase II y telofase II. Estos hallazgos avalan la desatención que sufren ciertos momentos y estructuras implicadas en la división celular, incluso en los libros de texto (Esquivel-Martín et al. 2021; Orcos y Magreñán 2018). Llegados a este punto, nos planteamos si la prometafase no se menciona en las pruebas EvAU porque los materiales educativos la omiten y los docentes no la enseñan; o si éstos no la enseñan porque no se pregunta en la EvAU.

En esta línea, Oliva, Franco-Mariscal y Gil (2018) señalan que la forma de evaluar es el criterio por excelencia de los profesores al tomar decisiones educativas, por lo que el diseño de la EvAU podría estar condicionando la actividad de los docentes en Bachillerato, programando el temario susceptible de ser preguntado en el examen para que los estudiantes obtengan buenos resultados. En consecuencia, nuestros hallazgos sugieren que las preguntas sobre división celular asociadas a una alta demanda cognitiva podrían ser infrecuentes en las aulas de Biología, al no ser necesarias para superar con éxito la EvAU. En este contexto, es importante considerar que solo cursan estudios universitarios un porcentaje limitado de alumnos (Vázquez et al. 2005), por lo que esta práctica docente podría condicionar una formación integral en ciencias para el resto del alumnado.

Además, el diseño de los exámenes EvAU, con preguntas recurrentes, también podría influir en la técnica de estudio empleada por el alumnado, decantándose por la memorización de respuestas tipo, ya que su meta es aprobar (Pozo 2018). Esto refleja un modelo de enseñanza-aprendizaje eficaz, pero no de calidad; que incumpliría con las exigencias de la etapa, distanciando a los estudiantes de una educación que los forme como ciudadanos críticos y alfabetizados. Evidencia de ello encontramos en el estudio de Balastegui et al. (2020), quienes señalan que los estudiantes del Bachillerato científico-tecnológico únicamente saben más conceptos científicos que los del Bachillerato de humanidades, pero no existen diferencias significativas en cuanto a sus actitudes hacia la ciencia, ni en la manera que tienen de abordar las relaciones ciencia-tecnologíasociedad. En consecuencia, y, a pesar de superar la EvAU, muchos alumnos llegan a carreras de ciencias con ideas alternativas, sin estar habituados a aplicar los conocimientos en contextos reales, y/o sin contar con capacidades esenciales en la universidad, como la autonomía en el aprendizaje (Falicoff, Domínguez y Odetti 2014; Rodríguez et al. 2018).

\section{CONCLUSIONES E IMPLICACIONES EDUCATIVAS}

Gracias al análisis realizado, se ha detectado que el conocimiento preuniversitario sobre división celular se está evaluando, sobre todo, con preguntas de relato y de producto; repitiéndose algunas de ellas con relativa frecuencia en los exámenes EvAU. Se ha observado una tendencia a aumentar las preguntas de producto hasta 2016, donde su frecuencia se iguala a las de proceso. Además de predominar las preguntas sobre mitosis de células animales, en estas pruebas se da excesivo protagonismo al material genético y a etapas como la anafase, en detrimento de otras estructuras y momentos del proceso igualmente esenciales, como el huso mitótico o la prometafase (Esquivel-Martín et al. 2021). Asimismo, la presencia de preguntas con errores y la ausencia de imágenes reales podrían estar dificultando el proceso de enseñanza-aprendizaje de la división celular.

En este contexto, si atendemos a los aspectos clave para el desarrollo de una adecuada alfabetización científica (Figura 1), el diseño de las preguntas de EvAU sobre división celular no permite determinar el grado 
de alfabetización científica alcanzado por el alumnado, ya que no integra la evaluación competencial ("saber hacer”). Así, aunque las preguntas del examen se adecúen a los escasos estándares de aprendizaje evaluables del currículo sobre división celular a nivel conceptual ("saber") (Real Decreto 1105/2014), ninguna de ellas demanda que los estudiantes tomen decisiones. Por ejemplo, valorando el impacto (ambiental, social, sanitario) de posibles soluciones para un problema dado. Al ser una prueba escrita puntual, tampoco permite evaluar el grado de compromiso social del alumnado. Por tanto, solo las preguntas de proceso van más allá de la alfabetización fundamental y de los conocimientos y competencias científicas básicas, al requerir una comprensión científica del contexto concreto sobre el que los alumnos deben aplicar el modelo de división celular.

Nuestros resultados, junto a los de Ferrés-Gurt (2017), contribuyen a establecer un marco de referencia del estado de la cuestión en España, sobre el cual basar las propuestas de mejora de los exámenes de Biología para acceder a la universidad. Y es que, plantear cambios pasa por ser conscientes de qué se considera suficiente para iniciar cualquier carrera científica y dónde está poniendo el foco el sistema de evaluación actual en Biología. Así, en primer lugar, consideramos que las evaluaciones deberían incluir más preguntas cuya demanda cognitiva sea elevada, que requieran procesar lo aprendido, utilizando la ciencia como proceso que fomenta la reflexión, y no como producto o saber acabado fácilmente memorizable.

En este sentido, existen propuestas de actividades de evaluación basadas en problemas reales sobre división celular que permitirían poner en juego destrezas científicas básicas, como la descripción de observaciones; comunicativas, como la representación y el uso de modelos; de investigación, como la emisión de hipótesis; e incluso técnicas, como el diseño de experiencias detallando el método a seguir o los instrumentos a utilizar (Pro 2013). Por ejemplo, la secuencia didáctica de Esquivel-Martín et al. (2021) demanda a los estudiantes desde discutir posibles soluciones para reducir la contaminación de un río por fertilizantes y pesticidas, que causa problemas reproductivos en poblaciones de distintas especies; hasta razonar en qué fase está una supuesta célula tumoral tratada con un fármaco en una micrografía (uso monopolar en prometafase), o vista desde un ángulo atípico (cromosomas metafásicos en vista polar). Estas actividades se integran en historias que abordan problemas científicos en contextos cotidianos, y requieren procesar toda la información disponible y construir nuevos relatos para responder, lo que puede resultar atractivo y mejorar el interés del alumnado por las ciencias.

En segundo lugar, sería conveniente evitar incluir esquemas inadecuados en la EvAU, ya que pueden ser utilizados por los docentes en las aulas de Bachillerato, transmitiendo ideas alternativas. Finalmente, entendemos que añadir la modalidad de examen práctico en estas pruebas, como se hace en las Olimpiadas de Biología, sería complicado, por los recursos humanos, temporales y materiales disponibles. No obstante, consideramos que la realización de la EvAU empleando ordenadores, laboratorios virtuales, etc., como ha ocurrido en las dos últimas ediciones de PISA (Rosales et al. 2020), permitiría variar la tradicional formulación de las actividades, y complementar algunas preguntas con el uso de simuladores. Gracias a ello, se podría demandar a los estudiantes buscar información para evaluar el rigor de una noticia, ajustar variables, o comprobar hipótesis diseñando experimentos adecuados. Creemos que el protagonismo de la argumentación en las respuestas elevaría la calidad de estas evaluaciones, al asemejarse a las estrategias utilizadas en investigación científica. Para ello, sería conveniente que el desarrollo de la alfabetización científica no se desligue de la alfabetización mediática en las aulas. Así, entre las recomendaciones del Consejo Europeo 8015/18, aparece la necesidad de conocer mejor las herramientas digitales, capacitando al alumnado para combatir los riesgos en línea como las noticias falsas (Alonso 2019). Aunque en este trabajo nos centramos en la relevancia de evaluar si el alumnado posee un conocimiento científico suficiente para hacerlo, cabe destacar que existen otros factores no-epistémicos que influyen en la ciudadanía al tomar decisiones sobre cuestiones socio-científicas controvertidas (creencias culturales, sociales, políticas, morales, religiosas, intereses personales, etc.) (Lederman, Antink y Bartos 2014). 
En el futuro, se podría ampliar la muestra de estudio con las preguntas sobre división celular de los exámenes EvAU de Biología de más comunidades autónomas españolas. También podrían analizarse las preguntas de las Olimpiadas de Biología u otras pruebas estandarizadas en relación con este contenido. Asimismo, sería conveniente profundizar en qué y cómo se pregunta en las aulas sobre división celular; valorando en qué medida el diseño de la EvAU y/o los materiales educativos disponibles condicionan realmente la praxis docente, incluyendo la evaluación. Para ello, consideramos que, desde la investigación educativa, sería interesante consensuar un sistema de clasificación de preguntas universal, válido para cualquier materia, aunque luego puedan pormenorizarse las subcategorías atendiendo a contenidos concretos, salvando así la limitación metodológica que encontramos al iniciar este trabajo.

\section{Agradecimientos}

Este trabajo ha sido realizado gracias al contrato de investigación predoctoral FPI-UAM (TEM), en el marco de dos proyectos de investigación I+D+i [PGC2018-096581-B-C22 (TEM \& BBT); EDU2017-82688-P (JMPM)] y de la Cátedra UNESCO de Educación para la Justicia Social (TEM, JMPM \& BBT).

\section{REFERENCIAS}

Alonso M. (2019) Fake News: desinformación en la era de la sociedad de la información. Ambitos. Revista Internacional de Comunicación 45, 29-52.

Balastegui M., Palomar R., Solbes J. (2020) ¿En qué aspectos es más deficiente la alfabetización científica del alumnado de Bachillerato? Revista Eureka sobre Enseñanza y Divulgación de las Ciencias 17 (3), 3302-3316.

Bloom B. S. (1956) Taxonomy of educational objectives, handbook I: The cognitive domain. New York: David McKay.

Bravo-Torija B., Gil M. J., Martínez B., Embid B. (2016) How, when and why? An analysis of primary students' science questions. In $11^{\circ}$ International Congress of European Reaserachers in Didiactics of Biology. Karlstads, Sweden.

Caballer M. J., Giménez I. (1993) Las ideas del alumnado sobre el concepto de célula al finalizar la educación general básica. Enseñanza de las ciencias 11 (1), 63-68.

Dikmenli M. (2010) Misconceptions of cell division held by student teachers in biology: A drawing analysis. Scientific Research and Essay 5 (2), 235-247.

Domènech J. (2016) “Drug Research”: una secuencia contextualizada de indagación sobre mitosis, cáncer y creación del conocimiento científico. Investigación en la escuela 88, 93-111.

Esquivel-Martín T., Bravo-Torija B, Pérez-Martín J.M. (2021) Solving a problem about cancer treatment: How does the use of the mitotic spindle model evolve during small group discussions? Journal of Biological Education. $\mathrm{h}$ ttps://bit.ly/3hgLzoY

Esquivel-Martin T., Pérez-Martín J.M, Bravo-Torija B (2021) The Use of Storytelling to Promote Literacy Skills in Biology Education: An Intervention Proposal. In M. D. Ramírez-Verdugo, B. Otcu-Grillman (Eds.), Interdisciplinary Approaches Toward Enhancing Teacher Education (pp. 155-177). USA: IGI Global.

Falicoff C. B., Domínguez J. M., Odetti H. S. (2014) Competencia científica de estudiantes que ingresan y egresan de la Universidad. Enseñanza de las ciencias 32 (3), 133-154.

Ferrés-Gurt C. (2017) La competència d'indagació i la seva avaluació en els estudiants de batxillerat (Tesis doctoral). Universidad Autónoma de Barcelona, Cataluña.

Franco-Mariscal R., Oliva J. M., Gil A. (2015) Análisis de contenido de las pruebas de acceso a la universidad en la asignatura de Química en Andalucía. Revista Eureka sobre Enseñanza y Divulgación de las Ciencias 12 (3), 456-474.

Furman M. G., Poenitz M. V., Podestá M. E. (2012) La evaluación en la formación de los profesores de Ciencias. Praxis y Saber 3 (6), 165-189. 
Tamara Esquivel-Martín, et al. ¿Qué evalúan las Preguntas sobre división celular en las Pruebas de...

García-González S. M., Furman M. G. (2014) Categorización de preguntas formuladas antes y después de la enseñanza por indagación. Praxis y Saber 5 (10), 75-91.

Jin H., Mikeska J. N., Hokayem H., Mavronikolas E. (2019) Toward coherence in curriculum, instruction, and assessment: A review of learning progression literature. Science Education 103 (5), 1206-1234.

Johnson S. B., Park H. S., Gross C. P., James B. Y. (2018) Complementary medicine, refusal of conventional cancer therapy, and survival among patients with curable cancers. JAMA oncology 4 (10), 1375-1381.

Lederman N. G., Antink A., Bartos S. (2014) Nature of science, scientific inquiry, and socio-scientific issues arising from genetics: A pathway to developing a scientifically literate citizenry. Science \& Education 23 (2), 285-302.

Lederman N. G., Lederman J. S. (2016) I read it on the Internet, it has to be true! Journal of Science Teacher Education 27, 795-798.

Mateos C., Llobet T. (2019) II Estudio sobre Bulos en Salud. https://bit.ly/2YVeA0R

Menoyo M. P. (2003) Evaluación de habilidades científicas en las PAU de biología. Alambique 37, 58-69.

Miralles P., Gómez C.J., Sánchez R. (2014) Dime qué preguntas y te diré qué evalúas y enseñas. Análisis de los exámenes de ciencias sociales en tercer ciclo de Educación Primaria. Aula Abierta 42 (2), 83-89.

National Research Council (NRC) (2013) Next Generation Science Standards: For States, By States. Washington DC: The National Academies Press.

Occelli M. E., García L., Valeiras N., Willging P. A. (2017) Animar la división celular (mitosis): una propuesta didáctica con la técnica de slowmation. Revista Eureka sobre Enseñanza y Divulgación de las Ciencias 14 (2), 398-409.

O'Connor C., Joffe H. (2020) Intercoder reliability in qualitative research: debates and practical guidelines. International Journal of Qualitative Methods 19. https://bit.ly/3yjOT9N

OECD (2019) PISA 2018 Assessment and Analytical Framework. Paris: OECD Publishing.

Oliva J. M., Franco-Mariscal R., Gil M. L. (2018) Influencia de las pruebas de acceso a la universidad en la metodología docente del profesorado de ciencias. Apice. Revista de Educación Cientifica 2 (1), 1-17.

Orcos L., Magreñán Á. A. (2018) The hologram as a teaching medium for the acquisition of STEM contents. International Journal of Learning Technology 13 (2), 163-177.

Pérez-Martín J. M. (2018) Un viaje en el tiempo por la alfabetización científica en España. Didácticas Especificas 18, 144-166.

Pérez-Martín J.M., Aquilino M. (2015) Nuevas estrategias en la enseñanza de la mitosis. En M. González, A. Baratas, A. Brandi (Eds.), III Jornadas sobre investigación y didáctica en ESO y Bachillerato (pp.199-210). Madrid: Editorial Santillana.

Pérez-Martín J. M., Calurano M., Martín-Aguilar C., Esquivel-Martín T., Bravo-Torija B. (2019) Preguntas en los libros de texto de Ciencias Naturales de Educación Primaria: ¿Procesando o reproduciendo contenidos? ReiDoCrea 8, 186-201.

Pozo J. I. (2018) ¿Por qué los alumnos no quieren aprender lo que les queremos enseñar? Desde la patagonia. Difundiendo saberes 15 (26), 4-7.

Pro A. J. (2013) Enseñar procedimientos: por qué y para qué. Alambique: Didáctica de las ciencias experimentales 73 , 69-76.

Quse L., Masullo M. S. (2017) ¿Las preguntas de biología en la evaluación nacional “Aprender-2016” de Argentina visibilizan el pensamiento? Enseñanza de las ciencias (Extra), 1289-1294.

Real Decreto 1105/2014, de 26 de diciembre, por el que se establece el currículo básico de la Educación Secundaria Obligatoria y del Bachillerato, BOE núm. 3 (2015).

Revesado D. (2018) El proceso de Bolonia y la admisión a la universidad. La realidad del sistema universitario español en perspectiva comparada. Revista Española de Educación Comparada 32, 169-180.

Roca M., Márquez C., Sanmartí N. (2013) Las preguntas de los alumnos: Una propuesta de análisis. Enseñanza de las Ciencias 31 (1), 95-114.

Rodríguez S. G., Fradkin M., Castañeda A. N. (2019) Conceptions of meiosis: misunderstandings among university students and errors. Journal of Biological Education 53 (2), 191-204. 
Rosales E. M., Rodríguez P. G., Romero-Ariza M. (2020) Conocimiento, demanda cognitiva y contextos en la evaluación de la alfabetización científica en PISA. Revista Eureka sobre Enseñanza y Divulgación de las Ciencias 17 (2), 2302-2323.

Sánchez J. (2004) Libro Blanco del Título de Grado en Biología. ANECA. España: Red de Biología.

Siarova H., Sternadel D., Szőnyi E. (2019) Research for CULT Committee - Science and Scientific Literacy as an Educational Challenge. Brussels: European Parliament, Policy Department for Structural and Cohesion Policies.

Smith K.C., Nakhleh M.B., Bretz S.L. (2010) An expanded Framework for analyzing general chemistry exams. Chemistry Education Research and Practice 11, 147-153.

Vázquez A., Acevedo J. A., Manassero M. A. (2005) Más allá de la enseñanza de las ciencias para científicos: hacia una educación científica humanística. Revista electrónica de Enseñanza de las Ciencias 4 (2), 1-30.

Vázquez Á., Manassero M. A. (2019) La educación de ciencias en contexto: Aportaciones a la formación del profesorado. Tecné, Episteme y Didaxis 46, 15-37.

Vieira R. M., Tenreiro-Vieira C. (2016) Fostering scientific literacy and critical thinking in elementary science education. International Journal of science and mathematics education 14 (4), 659-680.

Yore L. (2012) Science Literacy for All: More than a Slogan, Logo, or Rally Flag! In K.C.D. Tan, M. Kim (Eds.), Issues and Challenges in Science Education Research Moving Forward (pp. 5-23). London: Springer.

Zoller U., Lubesky A. Nakhleh M.B., Tessier B., Dori J. (1995) Success on algorithmic and LOCS vs. conceptual chemistry exam questions. Journal of Chemical Education 72, 987-989.

\section{INFORMACIÓN ADICIONAL}

Para citar este artículo: Esquivel-Martín T., Pérez-Martín J. M. y Bravo-Torija B. (2022) ¿Qué evalúan las preguntas sobre división celular en las pruebas de acceso a la universidad? Revista Eureka sobre Enseñanza y Divulgación de las Ciencias 19(1), 1104.doi: 10.25267/Rev_Eureka_ensen_divulg_cienc.2022.v19.i1.1104 